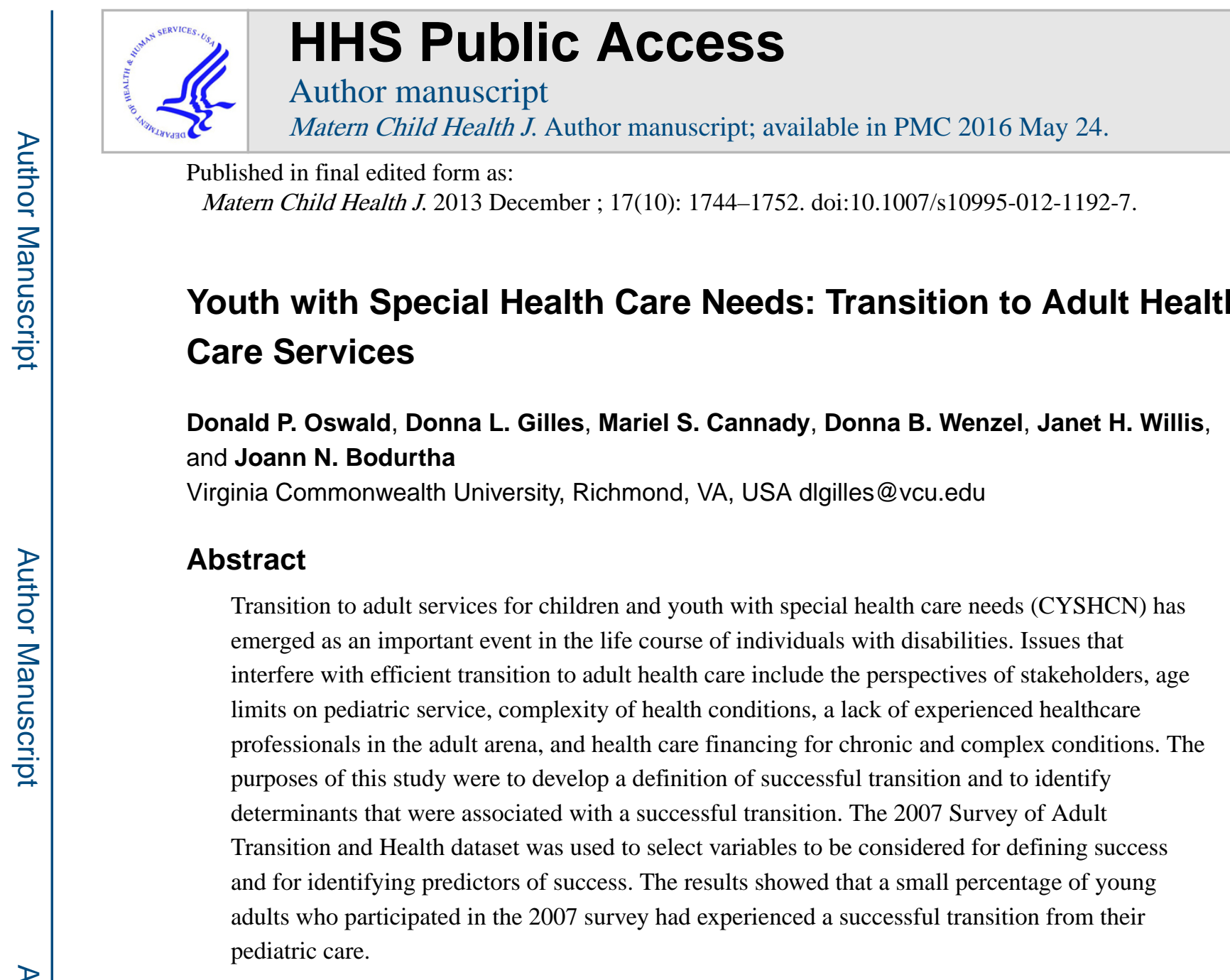

Keywords

Transition; CSHCN; CYSHCN; Pediatrics; Adult health; Disabilities

\title{
Overview and Objectives
}

The successful transition to adult health care services for children and youth with special health care needs (CYSHCN) is becoming a key area of concern as more people with disabilities are living well into their adult years. One of the six core outcomes for CYSHCN identified by the Maternal and Child Health Bureau was: "Youth with special health care needs receive the services necessary to make transitions to all aspects of adult life, including adult health care, work, and independence" [1]. Clearly, the transition to adult health care services is only one of several important transition issues, each of which deserves

\footnotetext{
D. P. Oswald

doswald@autismva.org

M. S. Cannady

marielcannady@me.com

D. B. Wenzel

momof11@embarqmail.com

J. H. Willis

jhorsley@vcu.edu

J. N. Bodurtha

jbodurtha@mcvh-vcu.edu
} 
investigation. However, medical advances that have extended life expectancy have made more salient the issue of transition to adult health care for individuals with genetic conditions such as cystic fibrosis (CF), chronic health conditions such as congenital heart disease and HIV-AIDS, and developmental disabilities accompanied by other health conditions.

The Cystic Fibrosis Foundation [2] has developed specialist clinical fellowships for physicians and mandated accredited adult centers when programs are serving 40 or more adults with CF. A recent study of transition practices in the CF Centers across the country identified great variability in services and supports for young adults involved in the process of health care transition. The survey yielded 170 responses from CF programs and indicated that less than half of these programs performed a transition readiness assessment, less than 25 percent of programs "usually" or "always" provided educational materials about transition to patients and families, and less than 10 percent had a written list of selfmanagement skills to support a successful transition [3].

Similarly, in a study of 260 adolescents and young adults with congenital heart disease, only 47 percent had successful transitions, and more than 25 percent had not seen a cardiologist since the age of 18 years [4]. These findings raised concerns about increased morbidity and long-term complications that can result from a lack of appropriate health care and follow-up for a complex medical condition. Dowshen and D'Angelo [5] discussed transition challenges for youth with HIV/AIDS and called for future research to study clinical outcomes of transition models in areas where resources were less available. The challenges of complex medical conditions and varying trajectories toward independence may result in specific health and social needs requiring adult networks that are not well developed. For example, young adults with autism spectrum disorders (ASD) show a high rate of disengaging from support services i.e., not receiving any medical, mental health, case management or speech therapy services, as reported by nearly 40 percent of parents [6]. Similarly, in reviewing models of transitional care for children with complex health needs including cerebral palsy, ASD, and diabetes, Watson et al. [7] discovered little evidence to inform practice and no models supporting successful transition of youth with ASD.

The challenges for CYSHCN in successfully transitioning to adult health care services are related to the perspectives of the stakeholders involved in the transition process, as well as to issues within the health care system. Schwartz et al. [8] described a social-ecological model of transition readiness on the part of the stakeholders, which included the patient and family, the pediatric providers, the adult health care providers, and others outside the patient's immediate social and medical circle. The authors further described transition as a dynamic process that is preceded by transition readiness, and improvement in readiness is dependent on good transition assessment and planning. One barrier to successful transition is the fear or ambivalence that adolescents and young adults may experience regarding the move from pediatric to adult care, which can interfere with or delay the transition process [9]. Similarly, adolescents, young adults, family members and health care providers have identified cognitive ability, maturity, and experience with personal responsibility for medical management as important considerations for the individual planning for transition [10]. 
Transition planning and implementation must be individualized with psychosocial support services for adolescents and young adults involved in the process [11].

Pediatric providers lack health outcome data regarding successful transition for specific conditions to support efforts to move to adult services [11]. Another obstacle is the more "disease-centered" approach of adult care, compared to the more comprehensive, patientcentered care often found in pediatric medical care [10]. This difference may lead to issues of trust or confidence in the provider of adult health care services.

Providers and practices serving adults also have important concerns about health care transitions for CYS-HCN. Peter et al. [12] surveyed internists regarding accepting chronically ill young adults into their practice. The top-rated concerns were medical competency to manage complex chronic conditions and to address adolescent health; the psychosocial issues related to a chronic illness, including end-of-life issues; the degree of family involvement and support for the chronically ill individual; increased demands for time and attention in the delivery of health care; and health care financing limitations and time constraints for patient care [12]. These and other concerns may threaten successful health care transition outcomes.

Larger system issues may create additional barriers to transition. Such issues include age limits for pediatric services, which force the issue of transition; regulations for public and private insurance programs (which may become less of a barrier with the advent of the Affordable Care Act of 2010); and the availability of trained and experienced providers to manage special health care needs and chronic conditions in the adult years [10].

In pursuit of the MCHB core outcome related to transition, the objectives of the present study were: (1) to construct a definition of successful transition to adult health care for CYSHCN, and (2) to identify characteristics of CYSHCN that were associated with an increased likelihood of a successful transition to adult health care services. This study examined only aspects of the health care transition; other no-less-important features of youth's transition to adulthood were not addressed in the survey and were thus outside the scope of the study.

\section{Methods}

\section{Survey}

The 2007 Survey of Adult Transition and Health (SATH) was designed as a national followback study to the 2001 National Survey of Children with Special Health Care Needs (NSCSHCN). A follow-back study is used to characterize individuals who achieved a specific outcome by identifying individual, family or larger system characteristics associated with that outcome. Both surveys were sponsored by the Maternal and Child Health Bureau (MCHB) of the U.S. Department of Health and Human Services, Health Resources and Services Administration. SATH collected current health status information from young adults aged 19-23 years who had been the subject of the detailed 2001 NS-CSHCN interview; these subjects were aged 14-17 years in 2001. In 2007, selected parents or guardians who completed 2001 NS-CSHCN interviews were contacted to obtain current, 
valid contact information for the 2001-sampled CSHCN, now young adults. With the assistance of the original 2001 parent or guardian respondent, the young adults were then contacted directly to invite them to participate in the 2007 SATH [13].

\section{Sample}

Target subjects for the SATH were 10,933 young adults who were 14-to-17 years old in 2001, had been identified as CSHCN, and lived in English-speaking households. According to survey documentation, this group was selected because in 2007 they would be legal adults capable of giving informed consent for their own interview and health care. In 2007, SATH surveyors were unable to contact 7,409 parents or guardians in the original target subject group. They successfully contacted and completed SATH interviews with 1,910 young adults, of whom 1,865 were included in the final dataset. Because the dataset did not include any information on individuals that were not successfully contacted, it was not possible to determine whether the present sample differed from the pool of potential subjects in any significant way. As a result, National Center for Health Statistics Division for Health Interview Statistics, the owner of the dataset, has suggested that the sample may be best described as a purposive or convenience sample rather than a random sample [13].

Cases were weighted so that the sample would represent the SATH target population of CSHCN in the United States aged 14-17 in 2001 who lived in English-speaking households, and who had completed a NS-CSHCN interview. Proxy interviews were conducted with the person who made the majority of the decisions about the young adult's health care for 113 young adults who were unable to complete the SATH interview on their own because of physical, cognitive, speech, or hearing difficulties.

Documentation for the 2007 SATH dataset indicated that sampling weights were derived from the original 2001 NS-CSHCN sampling weights and were then adjusted for nonresponse to the SATH interview, using demographic information from the 2001 NS-CSHCN. However, there was no analysis of non-response bias and young adults from households that did not move between 2001 and 2007 may be overrepresented in the dataset. Documentation acknowledges that these young adults may differ in important ways from young adults living in households that did move between 2001 and 2007.

\section{Selection of Variables}

The existing literature on transition to adult health care was first reviewed to assist in constructing a definition of what constitutes a successful transition. Results of this review were aligned with variables included in the SATH dataset to the extent possible, although some of the identified indicators of successful transition were not represented in the dataset (e.g., care involves a focus on the individual rather than the family, cited by Kennedy and Sawyer [14]). An initial pool of 24 survey items was selected for further consideration.

After further review, a number of potential variables was eliminated because (1) they were mostly missing due to the branching nature of the survey, or (2) they were judged to be duplicative or a specific instance of a more general variable already included in the definition. Following this review of the data, the pool was further narrowed to nine items (listed in Table 1) chosen to constitute a definition of a successful transition. An algorithm 
was devised to fine-tune the definition and deal with overlapping or possibly redundant items (i.e., a successful transition was defined to require that a young adult respondent have a usual health care source or a usual routine preventive care source or a personal doctor or nurse). Each of the remaining six items in the definition was required in order to meet our definition of a successful transition. Thus, according to this definition, a young adult has made a successful transition to adult health care services if he/she:

- had a usual health care source or personal doctor/nurse;

- had a health care provider who did not treat only children, teens, or young adults;

- had health insurance coverage that meets his/her needs;

- had at least one recent (within the past 12 months) preventive health care visit;

- was satisfied with health care services;

- had not recently (within the past 12 months) delayed or foregone needed health care services.

We next reviewed the literature again to identify child and family characteristics that might be expected to be predictors of successful transition and screened the original 2001 survey dataset for variables that captured those constructs. We initially identified a large number of potential predictors ( 98 variables); through a process of combining variables, eliminating variables with excessive missing data, and eliminating variables dropped from the regression models due to collinearity; the final pool of predictors was narrowed to 32 variables (see Table 2).

\section{Data Analysis}

Data analyses were conducted using the svyprop and svylogit procedures in the statistical analysis package, Stata 8.1. Use of these procedures allows for the generation of standard errors appropriate to the complex sample design. Logistic regression models were created using "successful transition (yes/no)" as the response variable.

Because of the large number of predictor variables, in the first stage of the predictors analysis, logistic regression models were constructed with logically grouped sets of predictors (see Table 2). 2001 Screener questions were included as potential predictors because they represent the indicators by which youth were entered into the NS-CSHCN sample, although the screener questions do not reflect a common underlying construct and they may overlap with other potential predictors.

In a second stage, significant predictors from stage 1 were combined into a final model to determine which of them made independent contributions to the prediction of successful transition.

\section{Results}

Based on our definition, 21.6 percent $(\mathrm{SE}=.014$ ) of youth made a successful transition to adult health care. Each of the individual indicators contributing to the definition of a successful transition was met by a majority of the youth in the sample (see Table 1) ranging 
from $62.6 \%$ ("had continuous health insurance coverage for the past 12 months") to $89.1 \%$ ("has usual health care source OR usual routine preventive care source OR personal doctor or nurse"). Approximately two-thirds of the sample (65.6\%) met four, five, or six of the seven indicators in the definition, while $12.1 \%$ met only zero to three indicators.

As an informal validity check on the definition, we explored whether making a successful transition to adult health care was associated with the young adult's health status at the follow-up interview. Young adults were asked whether their health in 2007 was "better", "about the same", or "worse" than in 2001. Young adults who had made a successful transition were significantly more likely to say their health was better or about the same, compared to those who had not made a successful transition $(\mathrm{OR}=2.3 ; \mathrm{t}=3.28 ; p=0.001$; $95 \% \mathrm{CI}=1.4-3.8)$.

In a small number of cases $(\mathrm{n}=113)$, survey data were based on proxy interviews to accommodate young adults who were unable to complete the SATH interview on their own because of physical, cognitive, speech, or hearing difficulties. Interviewers asked parent or caretakers who made the majority of the decisions about the young adult's health care to verify that the sampled person was unable to participate; in such cases the parents or other caregivers served as a proxy respondent for the SATH interview. Proxy respondents were told to answer the questions from the point of view of the young adult and were read the consent statement [13] (p. 4). Having a proxy as a respondent was not a significant predictor of whether the youth made a successful transition $(\mathrm{OR}=.94 ; p=.84 ; 95 \% \mathrm{CI}=.48-1.81)$. Thus, it did not appear that youth with significant physical, cognitive, speech, or hearing impairment were any more or less likely to transition successfully.

Stage 1 analyses yielded nine significant predictors of a successful transition (bolded variables in Table 2). These nine predictors were included in a final (Stage 2) model. The Stage 2 model was significant $(\mathrm{F}(15,1571)=2.00 ; p=.01)$ and results (see Table 3 ) indicated that youth were more likely to make a successful transition if, in 2001, they:

- had a mother with a college education;

- were female;

- received all the routine preventive care they needed in childhood;

- had insurance in childhood that allowed them to see the

- providers they needed;

- had doctors or other health care providers in childhood who listened carefully to them and to their parents,

\section{Conclusions}

Survey data suggested that the rate of successful transition to adult health care for CYSHCN was low. Only about one in five adolescents with special health care needs successfully transitioned to adult health care services. While further investigation of the proposed definition of a successful transition is needed, this finding raises significant concern that the 
health care system currently fails to provide the supports necessary for adolescents to ensure that their health care needs continue to be addressed as they leave pediatric care.

Results of the present study also suggest that there are individual characteristics, as well as features of the pediatric health care experience, that are associated with the likelihood of successful transition that may be useful in understanding the issue and designing a response.

\section{The Role of Maternal Education}

Maternal education is often used as a proxy for household socioeconomic status (SES, see for example Auger et al. [15]). However, given the fact that "Poverty level of the household" was included in the initial pool of predictor variables, it seems likely that maternal education plays some role above and beyond SES. Lykens, Fulda, Bae and Singh [16] reported that, for CSHCN, maternal education was associated with children receiving all needed specialist care for households at some income levels. They speculated that "women in the lower SES stratum who are also less educated may have less knowledge of which specialty services could benefit their special needs child and perhaps a lower expectation of the health care system" (p. 5). A similar explanation may apply to the present findings; mothers with lower education levels may be less able to provide their adolescent youth with information about the health care system that will allow them to transition successfully to adult care.

\section{Gender Differences}

Survey data suggest that young women are more likely to make a successful transition to adult health care than are young men. This finding is consistent with earlier reports that, for example, compared to adolescent males, adolescent females with depression are significantly more likely to utilize services as young adults [17]. Young adults who feel a greater need for health care services may be more likely to invest the effort required to connect with adult health care providers. Gender differences have been reported in individual characteristics that may affect transition to adult health care. Sawicki and colleagues [18] found that young adult females reported significantly higher self-advocacy skills than males on a transition questionnaire; however van Staa et al. [19] found that males reported more readiness for transition than females.

\section{Preventive Care}

The present study found that young adults who received preventive healthcare in adolescence were more likely to make a successful transition from pediatric to adult healthcare services than those youth whose preventive care was delayed or foregone. Yu et al. [20] reported that receiving preventive care was associated with age, insurance status, ethnicity, parent education, and child health status. Thus, it is possible that other predictors identified in the present study may contribute to the preventive care effect. However, receiving regular preventive care as an adolescent may also connect the youth with the health care system in a way that increases the likelihood of a successful transition to adult care.

Receiving preventive health care in adolescence may be an instance of a more general class of characteristics and experiences that serve to create positive connections between youth 
and the health care system and increase the likelihood of successful transition. Reid et al. [4] found only $27 \%$ of young adults with complex congenital heart disease (CHD) had any cardiac appointment at a Canadian Adult Congenital Heart (CACH) Network after the age of 18 years. They reported a successful transition was associated with a greater number of pediatric cardiovascular surgeries, at least one co-morbid medical condition, older age at the time of the last pediatric visit, medical record documentation of recommendations for follow up, and patient agreement that care should be obtained at a $\mathrm{CACH}$ center rather than with a general physician or general cardiologist. A successful transition was more likely if adolescents were provided with clear, simple instructions about where and when to receive adult care accompanied by referral letters and interim care given through transition clinics [4].

Additional factors associated with a successful transition in the Reid et al. [4] study were lack of excessive substance use, dental antibiotic prophylaxis, and attending cardiac appointments alone or with a peer rather than with a parent or sibling. Continued care during adolescence allows physicians to focus on risky health behaviors and provide appropriate treatment. The length of time spent under the care of the pediatric cardiologist during adolescence was associated with the likelihood of receiving a recommendation for adult health services. Singh et al. [21] found that having a severe and enduring mental illness, adherence with a medication regime, recent hospitalization, and continuity of care (at least one transition planning meeting with mental health personnel, and one or more parents in the weeks preceding transition) were factors found to be associated with a successful transition from child and adolescent psychiatry services to adult psychiatry services.

\section{Insurance}

Youth whose insurance coverage during childhood allowed them to see the providers they needed were more likely to make a successful transition. The implications of this finding are difficult to interpret with confidence. While it is possible that adequate insurance coverage is the "active ingredient" in this relationship, it is also possible that the survey item tapped into a more general quality indicator. If insurance allows a child to see the providers he/she needed, presumably the child received more adequate care, and it is not unreasonable to hypothesize that better quality care in childhood contributes to the likelihood of a successful transition to adult health care. Further investigation in this area may be able to disentangle these overlapping predictors, particularly if critical provisions of the Affordable Care Act of 2010 are allowed to take effect permanently. Barriers that have prevented youth with special health care needs from having access to health insurance will be reduced because of at least three key provisions: (1) pre-existing conditions cannot be a reason for denying them access; (2) young adults can stay on their parents' insurance plans until they are 26 years old; and (3) lifetime limits on claims will not be allowed. If having health insurance coverage is truly a predictor of successful transition, we can hypothesize that successful transitions will increase over the next five to ten years. Nonetheless, the health insurance factor must be studied closely in future surveys. 


\section{Listening Carefully}

There is little previous research regarding whether a provider who "listens carefully" is helpful in health care transitions. In addition, if an adolescent does not have adequate health insurance, there may be limited access to any professional, let alone one who "listens carefully".

Nonetheless, the overall conceptual framework for optimal transitions includes an effective support system for the child from family, friends, and, within the health care context, health care providers and institutions [22]. The importance of empathy and communication in the therapeutic encounter, in which listening is a key component, has been well documented [23]. In interviews and transition narratives, trust was a vital component of the relationships that young adults with disabilities had developed with their pediatric providers $[9,10]$. Others have indicated that improved communication with all providers, and whenever possible, a leadership role for the adolescent on the transition team, may help to ensure more comprehensive, less fragmented or redundant services [24].

The variation in health care delivery approaches to childhood and adult conditions involving disabilities (e.g. specialized clinics for cystic fibrosis and hemophilia) can differ significantly. Time in a health care encounter is likely to be shorter in adulthood [25]. Thus, it may be particularly important to consider time for listening in the transition plan.

Nurses and nurse practitioners can play an important role in the transition process both as careful listeners and as facilitators. Foster and Holmes [26] found that transition for adolescents with epidermolysis bullosa (EB) was facilitated by introducing the process at health care appointments beginning at age 12 with transfer to adult services between the ages of 16-18 years. Recognizing that parents and adolescents have many anxieties related to the transfer, EB nurses attended appointments, made home visits, and visited the adult EB center with the child and parents. Discussion of transition was accomplished gradually over multiple visits, and the adult-service EB nurse was a familiar figure when the adolescent completed the transition. In youth with congenital heart disease, Jalkut and Allen [27], found that the process was facilitated when pediatric nurse practitioners provided health education at each visit, discussed transition, accompanied the young adult to a pre-transfer visit in the adult CHD clinic, and ensured follow-up visits were scheduled.

\section{Limitations}

The generalizability of the present findings is limited in several important ways. As noted above, the survey Design and Operation manual concluded: "Because of the sample design, response rate, and the possibility of nonresponse bias, analysts may wish to consider the SATH sample as a convenience or purposive sample rather than a random sample" [13] (p. 11). Therefore, the results of this investigation are best viewed as useful for hypothesisgeneration rather than as testing any specific hypotheses.

In addition, the definition of a successful transition has not been independently validated. There may be important indicators of a successful transition that were overlooked in this survey or that were not addressed in the survey. Further conceptual and empirical work is needed to clarify what constitutes a successful transition. Such work might, for example, 
investigate whether the components selected for the present definition, alone or in combination, are empirically associated with more comprehensive health outcome indicators. Further, additional markers of successful transition, not included in the SATH dataset, might be identified and investigated.

\section{Implications}

The present findings suggest several implications with respect to improved clinical practice. The results add support to the conclusion that a therapeutic encounter characterized by thoughtful attention and careful listening is not only inherently valuable but also increases the likelihood that youth and adults will remain positively connected to the health care system. Future work in this area would benefit from qualitative research that would allow the field to hear the voices of young adults describing their experience with health care transition. Additional research in the area of gender differences could help inform how health care professionals may use gender-specific psychosocial approaches to facilitate the transition process.

Other findings suggest a need for particular attention to groups of youth who are at-risk for a failed transition. Maternal education can support health care access for young adults either through job-related health insurance or enhancing a family's capacity to navigate the complex system of transition supports. Youth whose parents have little education are likely to require additional support and guidance in order to successfully transition to adult health care.

Finally, although outside the control of practitioners, the importance of sustained health insurance coverage to support preventive health care and ongoing communication with health providers emphasizes the need for a coherent and comprehensive health insurance system, a goal not yet fully realized in the U.S.

\section{Acknowledgments}

The Data Source is the Centers for Disease Control and Prevention, National Center for Health Statistics (NCHS), State and Local Area Integrated Telephone Survey (SLA-ITS), Survey of Adult Transition and Health, 2007. All analyses, interpretations, or conclusions reached are attributed to the authors. NCHS and SLAITS are responsible only for the initial data. This article was funded, in part, by Project \# T73MC00040 from the Maternal and Child Health Bureau (Public Health Service Act, Section 399BB(e)[1](A), as amended by the Combatting Autism Act of 2006), Health Resources and Services Administration, Department of Health and Human Services (2010-2011).

\section{References}

1. U.S. Department of Health and Human Services. The National Survey of Children with Special Health Care Needs Chartbook 2005-2006. U.S. Department of Health and Human Services; Rockville, Maryland: 2008. Health Resources and Services Administration, Maternal and Child Health Bureau.

2. Cystic Fibrosis Foundation. http://www.cff.org.

3. McLaughlin SE, Diener-West M, Indurkhya A, Rubin H, Heckmann R, Boyle MP. Improving transition from pediatric to adult cystic fibrosis care: Lessons from a national survey of current practices. Pediatrics. 2008; 121(5):e1161-e1166.

4. Reid GJ, Irvine MJ, McCrindle BW, Sananes R, Ritvo PG, Siu SC, et al. Prevalence and correlates of successful transfer from pediatric to adult health care among a cohort of young adults with complex congenital heart defects. Pediatrics. 2004; 113(3):e197-e205. [PubMed: 14993577] 
5. Dowshen N, D'Angelo L. Health care transition for youth living with HIV/AIDS. Pediatrics. 2011; 128(4):762-771. [PubMed: 21930548]

6. Shattuck PT, Wagner M, Narendorf S, Sterzing P, Hensley M. Post-high school service use among young adults with an autism spectrum disorder. Archives of Pediatrics and Adolescent Medicine. 2011; 165(2):141-146. doi:10.1001/archpediatrics. 2010.279. [PubMed: 21300654]

7. Watson R, Parr JR, Joyce C, May C, LeCouteur AS. Models of transitional care for young people with complex health needs: A scoping review. Child: Care, Health and Development. 2011; 37(6): 780-791.

8. Schwartz LA, Tuchman LK, Hobbie WL, Ginsberg JP. A social-ecological model of readiness for transition to adult-oriented care for adolescents and young adults with chronic health conditions. Child: Care, Health and Development. 2011; 37(6):883-895.

9. Tuchman LK, Slap GB, Britto MT. Transition to adult care: Experiences and expectations of adolescents with chronic illness. Child: Care, Health and Development. 2008; 34(5):557-563.

10. Reiss JG, Gibson RW, Walker LR. Health care transitions: Youth, family, and provider perspectives. Pediatrics. 2005; 115(1):112-120. [PubMed: 15629990]

11. Tuchman LK, Schwartz LA, Sawicki GS, Britto MT. Cystic fibrosis and transition to adult care. Pediatrics. 2010; 125(3):566-573. [PubMed: 20176665]

12. Peter NG, Forke CM, Ginsburg KR, Schwartz DF. Transition from pediatric to adult care: Internists' perspectives. Pediatrics. 2009; 123(2):417-423. [PubMed: 19171604]

13. O'Connor KS, Brooks KS, Nysse-Carris KL, Skalland BJ, Sipulski ET, Costanzo DM. Design and operation of the survey of adult transition and health, 2007. National Center for Health Statistics. Vital Health Stat. 2011; 1(52):1-85.

14. Kennedy A, Sawyer S. Transition from pediatric to adult services: Are we getting it right? Curr Opinion in Pediatrics. 2008; 20(4):403-409.

15. Auger N, Authier MA, Martinez J, Daniel M. The association between rural-urban continuum, maternal education and adverse birth outcomes in Québec, Canada. Journal of Rural Health Fall. 2009; 25(4):342-351.

16. Lykens KA, Fulda KG, Bae S, Singh KP. Differences in risk factors for children with special health care needs (CSHCN) receiving needed specialty care by socioeconomic status. BMC Pediatrics. 2009; 9:48. doi:10.1186/1471-2431-9-48. [PubMed: 19646227]

17. Yu JW, Adams SH, Burns J, Brindis CD, Irwin CE Jr. Use of mental health counseling as adolescents become young adults. Journal of Adolescent Health. 2008; 43(3):268-276. [PubMed: 18710682]

18. Sawicki GS, Lukens-Bull K, Yin X, Demars N, Huang I, Livingood W, et al. Measuring the transition readiness of youth with special healthcare needs: Validation of the TRAQ - transition readiness assessment questionnaire. Journal of Pediatric Psychology. 2011; 36(2):160-171. [PubMed: 20040605]

19. van Staa A, Van der Stege HA, Jedeloo S, Moll HA, Hilberink SR. Readiness to transfer to adult care of adolescents with chronic conditions: Exploration of associated factors. Journal of Adolescent Health. 2011; 48(3):295-302. [PubMed: 21338902]

20. Yu SM, Bellamy HA, Kogan MD, Dunbar JL, Schwalberg RH, Schuster MA. Factors that influence receipt of recommended preventive pediatric health and dental care. Pediatrics. 2002; 110(6):e73. [PubMed: 12456940]

21. Singh SP, Paul M, Ford T, Kramer T, Weaver T, McLaren S, et al. Process, outcome and experience of transition from child to adult mental healthcare: Multiperspective study. British Journal of Psychiatry. 2010; 197(4):305-312. doi:10.1192/bjp.bp.109.075135. [PubMed: 20884954]

22. Betz CL. Parent-professional partnerships: Bridging the disparate worlds of children, families, and professionals. Journal of Pediatric Nursing. 2006; 21(5):333-336. [PubMed: 16980133]

23. Griffin SJ, Kinmouth A, Veltman MWM, Gillard S, Grant J, Stewart M. Effect on health-related outcomes of interventions to alter the interaction between patients and practitioners: A systematic review of trials. Annals of Family Medicine. 2004; 2(6):595-608. [PubMed: 15576546]

24. Callahan ST, Winitzer RF, Feinstein MS, Keenan P. Transition from pediatric to adult-oriented health care: A challenge for patients with chronic disease. Current Opinion in Pediatrics. 2001; 13(4):310-316. [PubMed: 11717554] 
25. Grosse SD, Schechter MS, Kulkarni R, Lloyd-Puryear MA, Strickland B, Trevathan E. Models of comprehensive multidisciplinary care for individuals in the United States with genetic disorders. Pediatrics. 2009; 123(1):407-412. doi:10.1542/peds.2007-2875. [PubMed: 19117908]

26. Foster L, Holmes Y. Transition from paediatric to adult service in epidermolysis bullosa. British Journal of Nursing. 2007; 16(4):244-246. [PubMed: 17363858]

27. Jalkut MK, Allen PJ. Transition from pediatric to adult health care for adolescents with congenital heart disease: A review of the literature and clinical implications. Pediatric Nursing. 2009; 35(6): 381-387. [PubMed: 20166468] 


\section{Table 1}

Definition of a successful transition

A young adult has made a successful transition to adult health care services if he/she:
HAS USUAL HEALTH CARE SOURCE
OR
HAS USUAL ROUTINE PREVENTIVE CARE SOURCE
OR
HAS A PERSONAL DOCTOR OR NURSE) [Percent meeting $=89.1 ; \mathrm{SE}=0.01$ ]
AND
(His/her) DOCTOR (does not) TREAT ONLY CHILDREN, TEENS, OR YOUNG ADULTS [Percent meeting $=74.1 ; \mathrm{SE}=0.02]$
AND
(He/she has had continuous) HEALTH INSURANCE COVERAGE (for the past 12 months) [Percent meeting $=62.6 ; \mathrm{SE}=0.02]$
AND
HEALTH INSURANCE BENEFITS MEET (his/her) NEEDS [Percent meeting $=66.7 ;$ SE $=0.02]$
AND
(He/she had at least 1) PREVENTIVE (health) CARE VISIT (in the last 12 months) [Percent meeting $=78.5 ; \mathrm{SE}=0.02]$
AND
(He/she is satisfied) WITH HEALTH SERVICES [Percent meeting = 88.9; SE = 0.01]
AND
(Needed) HEALTH CARE (was not) DELAYED/FOREGONE (in the last 12 months) [Percent meeting $=71.1 ; \mathrm{SE}=0.02$ ]

Text in ALL CAPS was taken from the label for the corresponding variable in the data set 


\section{Table 2}

\section{Survey Items investigated as potential predictors of successful transition}

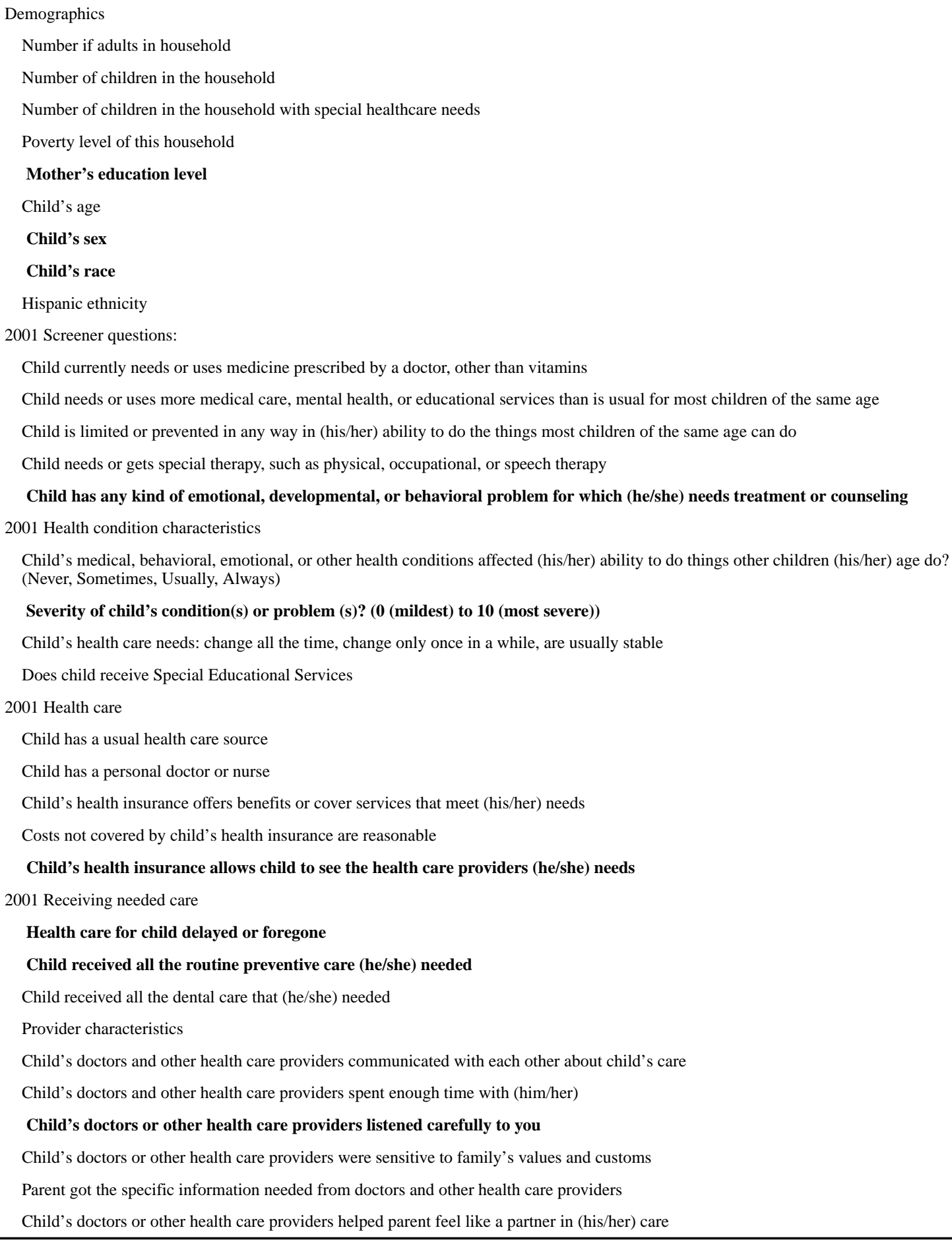

Child's health insurance allows child to see the health care providers (he/she) needs

2001 Receiving needed care

\section{Health care for child delayed or foregone}

Child received all the routine preventive care (he/she) needed

Child received all the dental care that (he/she) needed

Provider characteristics

Child's doctors and other health care providers communicated with each other about child's care

Child's doctors and other health care providers spent enough time with (him/her)

\section{Child's doctors or other health care providers listened carefully to you}

Child's doctors or other health care providers were sensitive to family's values and customs

Parent got the specific information needed from doctors and other health care providers

Child's doctors or other health care providers helped parent feel like a partner in (his/her) care

Variables in BOLD print were significant predictors at stage 1 and were combined in a stage 2 logistic regression model 


\section{Table 3}

\section{Predictors of successful transition}

\begin{tabular}{|c|c|c|c|c|c|}
\hline Predictor & Odds ratio & Std. Err. & $\mathbf{t}$ & $p$ & $95 \% \mathrm{CI}$ \\
\hline Mother's education level—graduated from college & 9.31 & 10.46 & 1.98 & 0.047 & $1.03-84.42$ \\
\hline Child's sex-female & 1.49 & 0.26 & 2.25 & 0.024 & $1.05-2.10$ \\
\hline Child's health insurance allows child to see the health care providers (he/she) needs & 2.42 & 0.82 & 2.61 & 0.009 & $1.25-4.71$ \\
\hline Child received all the routine preventive care (he/she) needed & 9.05 & 7.70 & 2.59 & 0.010 & $1.70-48.07$ \\
\hline Child's doctors or other health care providers listened carefully to you & 2.42 & 0.90 & 2.38 & 0.017 & $1.17-5.01$ \\
\hline
\end{tabular}

